\title{
Hybrid Management of a Pseudoaneurysm of the Inferior Gluteal Artery Following a Stab Wound
}

\author{
Imtinene Ben Mrad (iD) \\ Ramy Ben Salah ${ }^{2}$ \\ Melek Ben Mrad (iD ${ }^{3}$ \\ Rim Miri ${ }^{3}$ \\ Anis $\mathrm{Haddad}^{4}$ \\ Sobhi Mleyhi ${ }^{3}$ \\ Ihsen Zairi (1D) \\ Khalil Hamza ${ }^{5}$ \\ Mariem Jrad ${ }^{5}$ \\ Raouf Denguir ${ }^{3}$ \\ 'Cardiology Department, Hbib Thameur \\ Hospital, Tunis, Tunisia; ${ }^{2}$ Plastic Surgery \\ Department, Bizerte Hospital, Tunis, \\ Tunisia; ${ }^{3}$ Cardiovascular Surgery \\ Department, Rabta Hospital, Tunis, \\ Tunisia; ${ }^{4}$ General Surgery Department, \\ Rabta Hospital, Tunis, Tunisia; ${ }^{5}$ Radiology \\ Department, Charles Nicolle Hospital, \\ Tunis, Tunisia
}

\begin{abstract}
Aneurysms and pseudoaneurysms of the gluteal artery are rare. They represent less than $1 \%$ of the described arterial aneurysms. Those that touch the inferior gluteal artery are even rarer. Only a few cases have been described worldwide. Such cases often present with a variable time course, mode of injury, and associated symptoms, leading to their misdiagnosis and improper treatment. We present the case of a 30 -year-old male, who presented to our emergency room one week after a stab wound in the left gluteal region causing a pseudoaneurysm of the left inferior gluteal artery with a sciatic compartment syndrome treated by a hybrid approach.
\end{abstract}

Keywords: pseudoaneurysm, inferior gluteal artery, stab wound, embolization, sciatic nerve

\section{Introduction}

Aneurysms and pseudoaneurysms of the gluteal artery are rare. They represent less than $1 \%$ of the described arterial aneurysms. ${ }^{1}$ Those that touch the inferior gluteal artery are even more rare. Only a few cases have been described worldwide. ${ }^{2} \mathrm{We}$ present the case of a 30-year-old male, who presented to our emergency room one week after a stab wound in the left gluteal region causing a pseudoaneurysm of the left inferior gluteal artery with a sciatic compartment syndrome, that we treated by a hybrid approach.

\section{Observation}

We share the case of a 30 -year-old male, who is arrived in our emergency room with pain and swelling of the left gluteal region. The patient revealed that one week earlier he had been the victim of a stab wound in the left gluteal region. This wound was superficially sutured at a regional hospital, and the patient was then discharged.

The physical exam found swelling of the entire left gluteal region with blueblack blood coming out of the scar. Also, a $2 \mathrm{~cm}$ cutaneous necrosis at the superior part of the left gluteal region was noticed along with a splitting of surgical suture lines (Figure 1). Neither a pulsatile mass nor a thrill was detected on palpation of the right buttock. All the peripheral pulses were present and symmetrical. A complete blood count (CBC) found hemoglobin at $8.7 \mathrm{~g} / \mathrm{dL}$.

The patient also complained of left foot paresthesia. He also presented with total functional impotence of the left limb, including paresis of the toes and forefoot. The diagnosis of a sciatic compartment syndrome was retained.

We carried out a CT angiography of the aorta and the lower limbs that showed a voluminous hematoma infiltrating the gluteal muscles (Figure 2). The CT also
Correspondence: Melek Ben Mrad Cardiovascular Surgery Department, Rabta Hospital, App 2b, Bloc 12, Cite Olympique, Tunis 1003, Tunisia $\mathrm{Tel}+21655657116$

Email benmradmelek@yahoo.fr 


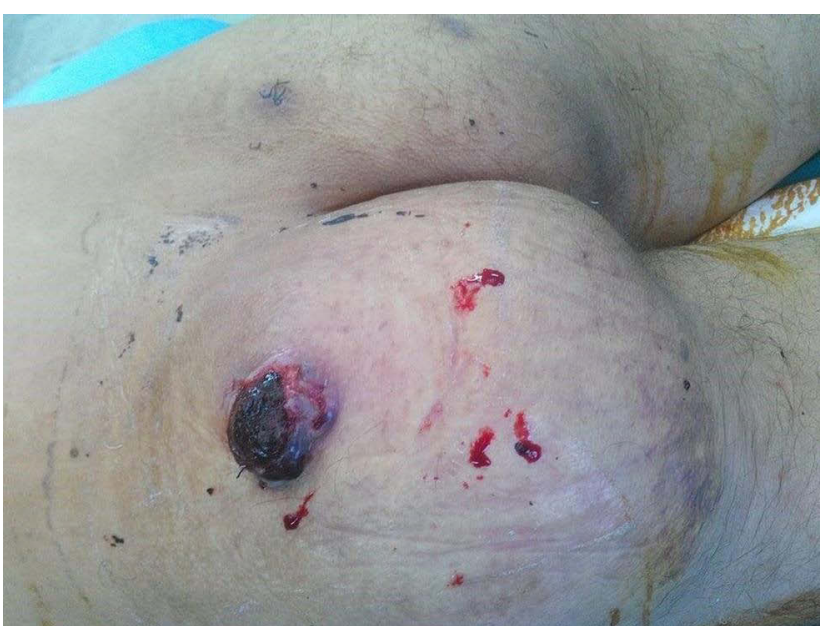

Figure I Swelling of the left gluteal region with $2 \mathrm{~cm}$ cutaneous necrosis at the superior part of the left gluteal region.

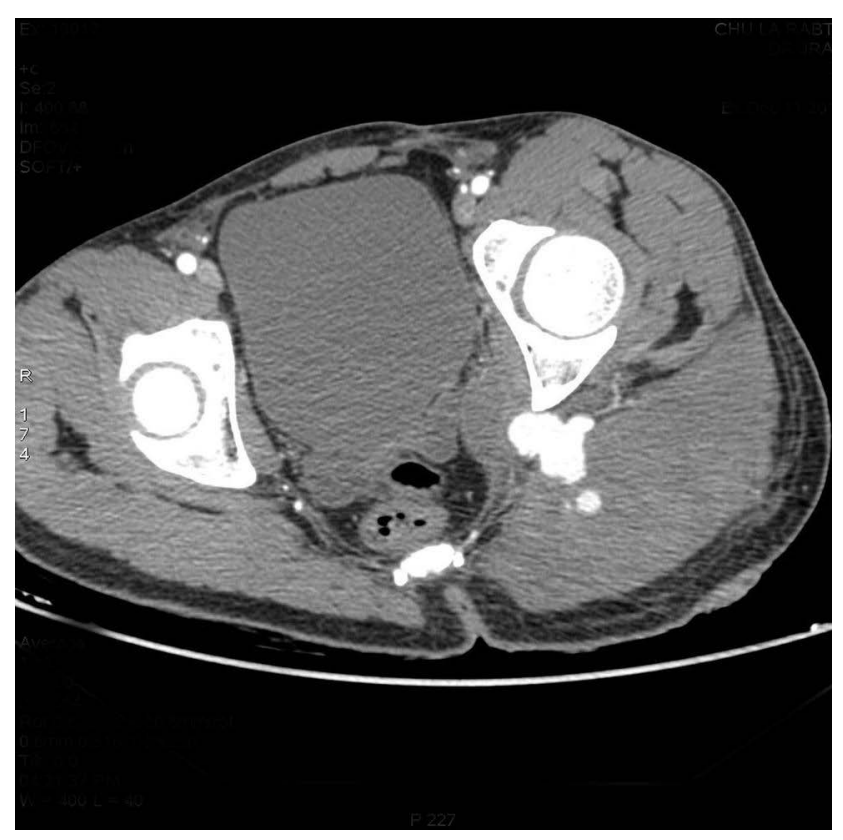

Figure 2 Axial CT scan showing voluminous hematoma infiltrating the gluteal muscles.

detected a $2 \mathrm{~cm}$ diameter pseudoaneurysm of the inferior gluteal artery situated between the quadratus femoris muscle and the gluteus maximus muscle (Figure 3).

According to the clinical and radiological presentation, we assumed that there was a high risk of rupture and hemorrhage, as well as a risk of irreversible nerve damage caused by the compression of the sciatic nerve. For this, we decided to intervene urgently.

First, the patient was taken to a hemodynamic suite. After a right groin retrograde access, a $45 \mathrm{~cm}$ cross-over $6 \mathrm{~F}$ sheath was placed into the left common iliac artery

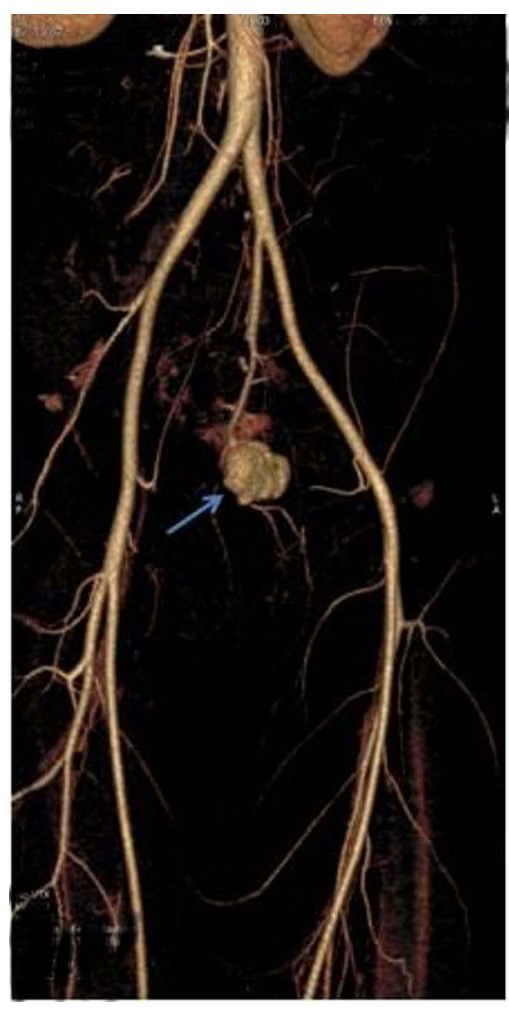

Figure 3 CT scan reconstruction showing pseudoaneurysm of the inferior gluteal artery (blue arrow).

using an UF catheter. The first angiogram confirmed the diagnosis of pseudoaneurysm of the gluteal artery (Figure 4). After a selective catheterization of this latter,

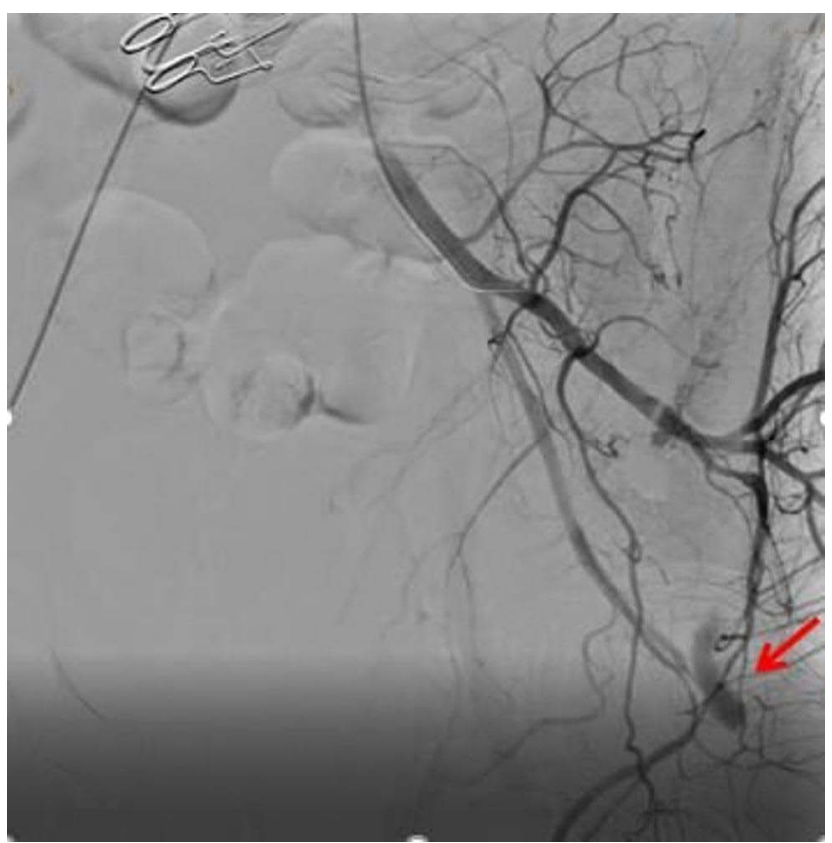

Figure 4 Angiography of the left internal iliac artery by a Bernstein catheter showing a pseudoaneurysm of the gluteal artery (red arrow). 
we deployed five $4 \mathrm{~mm}$ detachable coils allowing for the embolization of the gluteal artery (Figure 5).

The next day, in order to relieve the nerve compression and to avoid septic complications, the patient was operated on, then put in a prone position. He then had a gluteal hematoma evacuation, with a large debridement of the necrotic muscle fibres (Figure 6).

There was immediate improvement of gluteal pain and paraesthesia. No postoperative complications were reported. A double antibiotic treatment was prescribed for two weeks. A complete wound healing was obtained after a one-month period. After several rehabilitation sessions, the patient regained normal limb motor skills without neurological sequelae. After five years of clinical follow-up, the patient is well.

\section{Discussion}

The inferior gluteal artery is the largest terminal branch of the anterior segment of the internal iliac artery. ${ }^{2}$

Gluteal artery pseudoaneurysms are still uncommon. Fewer than 150 cases of pseudoaneurysms of the gluteal artery have been described in the literature to date. ${ }^{3}$ The Superior gluteal artery is more commonly hit. ${ }^{4}$ Pseudoaneurysms of the inferior gluteal artery after blunt trauma are even rarer, with just six cases reported in the literature. ${ }^{5}$

These pseudoaneurysms might appear after penetrating wounds of the gluteal region and hip fractures or might be iatrogenic and appear after an injection, a bone marrow transplant, or a hip replacement. ${ }^{1,2}$

The timeline for developing these pseudoaneurysms might range from a few weeks to a few years after the initial trauma. ${ }^{6}$

The clinical presentation is polymorphic. Patients might present with pain in the gluteal region, sciatica when the aneurysm is large, a gluteal mass, or even a life-threatening rupture. $^{7}$

This occurrence with sciatic nerve palsy caused by inferior gluteal artery laceration and compressive haematoma has been reported also by Rocos and Ward. ${ }^{8}$ To the best of our knowledge, this is the second time such a case has been reported in the literature.

The main differential diagnoses are; gluteal abscesses and gluteal tumours (lipoma, sarcoma). ${ }^{7}$

For every gluteal mass, the diagnosis of gluteal pseudoaneurysm should be also considered, and the physician should look for the notion of a trauma preceding the appearance of the mass. This diagnosis should be brought up even if the mass is not pulsatile upon clinical examination. ${ }^{8}$ Pulsation is an inconstant sign that could easily disappear if there is a small defect, a partial rupture of the wall, or the false aneurysm is completely lined by thrombus. ${ }^{9}$

Proceeding to an open repair of the mass with no further imaging might lead to catastrophic consequences up to and including uncontrollable hemorrhage compromising the vital prognosis of the patient. ${ }^{10}$

As soon as the diagnosis is suspected, a US duplex should be performed, which can show arterial flow within the hematoma interior and can differentiate other types of soft tissue injuries. ${ }^{1,3}$ Then, a CT angiography is necessary for a better visualization of the aneurysm and its anatomical relation enabling an adequate treatment plan to be put in place. ${ }^{11}$ In the present case, the patient underwent CT scan because he was hemodynamically stable.

For a long time, the management of these aneurysms has been based on open repair surgery. After a retroperitoneal approach and ligation of the hypogastric artery, the inferior gluteal artery is approached directly through a posterior approach. But this surgery is challenging, needing a dissection between the gluteal maximus

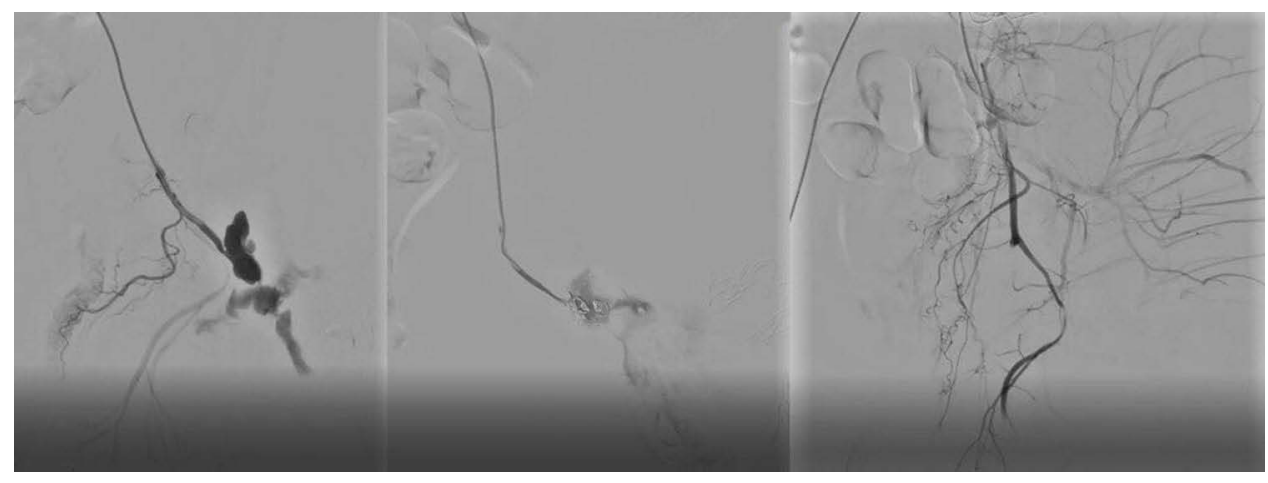

Figure 5 Superselective catheterization of the gluteal artery and occlusion of this latter by detachable coils. 


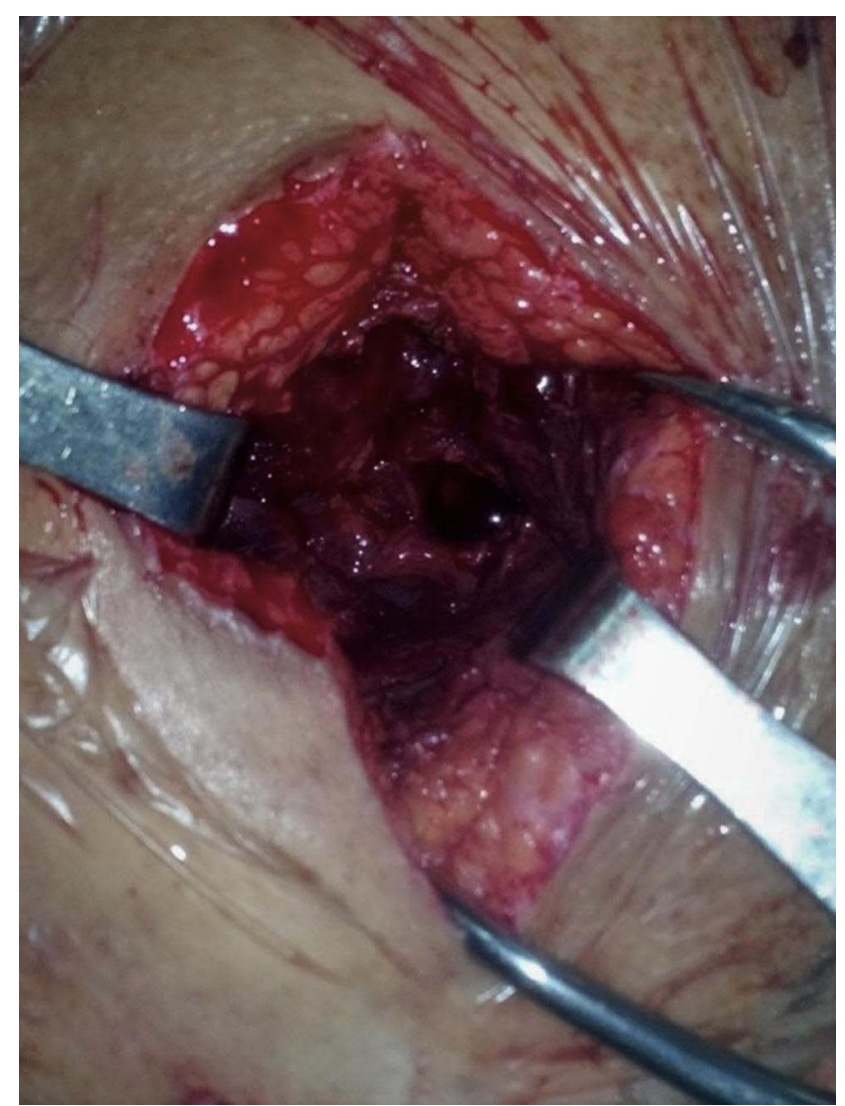

Figure 6 Preoperative image in prone position of the buttock showing a huge hematoma with muscle necrosis.

muscle and the gluteus medius muscle, exposing the sciatic nerve to potential lesions and the muscles to necrosis, with a high risk of uncontrollable haemorrhage. ${ }^{12}$

We thought that the most adequate method is to proceed by a hybrid approach in this case.

Starting first with a super selective embolization of the gluteal artery to stop the bleeding, the gluteal region can then be approached safely for a surgical evacuation of hematoma and debridement of tissue.

The percutaneous embolization technique, using coils to occlude the pseudoaneurysms represents an excellent alternative. ${ }^{13}$. Alternatives to coil embolization include embolization with glue or other emboligenic agents, such as cyanoacrylate glue and onyx. ${ }^{14}$

Compared to open repair, the embolization technique offers several advantages: it is a less complicated technique; it exposes the patient to lower risk of infection and hematoma, and lower risk of iatrogenic injuries to nerves or vessels; and it also allows the surgeon to avoid the retroperitoneal approach. ${ }^{2}$

However, its efficacy is reduced in large aneurysms. ${ }^{15}$
In the present case, emergency embolization successfully stopped the bleeding, avoiding potential permanent damage to the sciatic nerve by compression caused by the expanding gluteal hematoma. A surgical complement was necessary for the evacuation of this hematoma and to prevent septic complications.

\section{Conclusion}

Even if it is a rare occurrence, pseudoaneurysms of the gluteal inferior artery should be brought up and thought of in front of every patient presenting with a gluteal mass. Proceeding directly to a surgical repair, with no prior diagnosis, could expose patients to risk of incontrollable haemorrhage that could be potentially fatal.

Conventional surgical treatment is very challenging. The embolization technique is playing an increasing role in the treatment of these pseudoaneurysms; however, conventional surgery is still indicated in some cases, especially for the evacuation of possible hematomas.

\section{Institutional Approval}

We have the approval of our institution to publish the case details.

\section{Consent}

The patient has provided written informed consent to publish their case details and any accompanying images.

\section{Disclosure}

The authors report no conflicts of interest in this work.

\section{References}

1. Saad PF, Saad KR, Armstrong DM, Soares BL, de Almeida PH, Razuk Filho Á. Inferior gluteal artery pseudoaneurysm related to intramuscular injection. Int $J$ Surg Case Rep. 2015;6C:29-32. PMID: 25506847; PMCID: PMC4334878. doi:10.1016/j.ijscr.2014.10.080

2. Costa RF, Yoshida RA, Gibin RJ, et al. Inferior gluteal artery pseudoaneurysm after fall from a bicycle: case report. J Vasc Bras. 2018;17 (4):353-357. PMID: 30787957; PMCID: PMC6375272. doi:10.1590/ 1677-5449.003018

3. Corbacioglu KS, Aksel G, Yildiz A. Ruptured superior gluteal artery pseudoaneurysm with hemorrhagic shock: case report. Turk J Emerg Med. 2016;16(1):26-28. PMID: 27239635; PMCID: PMC4882201. doi:10.1016/j.tjem.2015.10.002

4. Demetriades D, Rabinowitz B, Sofianos C. Gluteal artery aneurysms. Br J Surg. 1988;75(5):494. PMID: 3390689. doi:10.1002/bjs. 180075 0532

5. Babu A, Gupta A, Sharma P, Ranjan P, Kumar A. Blunt traumatic superior gluteal artery pseudoaneurysm presenting as gluteal hematoma without bony injury: a rare case report. Chin J Traumatol. 2016;19(4):244-246. PMID: 27578385; PMCID: PMC4992137. doi:10.1016/j.cjtee.2015.11.018 
6. Khera G, Shea AJ, Parkinson R, Lambrianides AL. Delayed diagnosis of a gluteal pseudoaneurysm caused by blunt trauma: review of the literature and presentation of a case report. J Trauma. 2006;60 (3):644-647. PMID: $16531869 . \quad$ doi:10.1097/01. ta.0000204937.60174.84

7. Agarwal M, Giannoudis PV, Syed AA, Hinsche AF, Matthews SJ, Smith RM. Pseudoaneurysm of the inferior gluteal artery following polytrauma: diverse presentation of a dangerous complication: a report of two cases. J Orthop Trauma. 2003;17(1):70-74. PMID: 12499973. doi:10.1097/00005131-200301000-00013

8. Rocos B, Ward A. Gluteal compartment syndrome with sciatic nerve palsy caused by traumatic rupture of the inferior gluteal artery: a successful surgical treatment. BMJ Case Rep. 2017;2017 bcr2016216709. PMID: 28122800; PMCID: PMC5278333. doi:10.1136/bcr-2016-216709

9. Mikulin T, Walker EW. False aneurysm following blunt trauma Injury. 1984;15(5):309-310. PMID: 6706390. doi:10.1016/00201383(84)90052-4

10. Gilroy D, Saadia R, Hide G, Demetriades D. Penetrating injury to the gluteal region. J Trauma. 1992;32(3):294-297. PMID: 1548716. doi:10.1097/00005373-199203000-00005
11. Bennett JD, Brown TC, Coates CF, MacKenzie D, Sweeney J. Pseudoaneurysm of the inferior gluteal artery. Can Assoc Radiol J. 1992;43(4):296-298. PMID: 1638430.

12. Schorn B, Reitmeier F, Falk V, Oestmann JW, Dalichau H, Mohr FW. True aneurysm of the superior gluteal artery: case report and review of the literature. J Vasc Surg. 1995;21(5):851-854. PMID: 7769744. doi:10.1016/s0741-5214(05)80017-5

13. Mouawad NJ, Haurani MJ, Mason T, Satiani B. Delayed presentation and management of blunt traumatic inferior gluteal artery pseudoaneurysm with associated arteriovenous fistula. Vasc Endovascular Surg. 2013;47(7):573-576. PMID: 23883787. doi:10.1177/ 1538574413497272

14. Keeling AN, Naughton PA, Leahy AL, Lee MJ. Traumatic inferior gluteal artery pseudoaneurysm and arteriovenous fistula managed with emergency transcatheter embolization. Cardiovasc Intervent Radiol. 2008;31(Suppl 2):S135-9. PMID: 17710471. doi:10.1007/ s00270-007-9150-2

15. Herber SC, Ajalat GM, Smith DC, Hinshaw DB Jr, Killeen JD Jr. Transcatheter embolization facilitating surgical management of a giant inferior gluteal artery pseudoaneurysm. J Vasc Surg. 1988;8 (6):716-720. PMID: 3193550. doi:10.1067/mva.1988.avs0080716

\section{Publish your work in this journal}

The Open Access Emergency Medicine is an international, peerreviewed, open access journal publishing original research, reports, editorials, reviews and commentaries on all aspects of emergency medicine. The manuscript management system is completely online and includes a very quick and fair peer-review system, which is all easy to use. Visit http://www.dovepress.com/testimonials.php to read real quotes from published authors. 